\title{
Observation of the optical analogue of the quantised conductance of a point contact
}

\author{
E.A. Montie, E.C. Cosman, G.W. 't Hooft, M.B. van der Mark and C.W.J. Beenakker \\ Philps Research Laboratories, P.O. Box 80000, 5600 JA Eindhoven. The Netherlands
}

\begin{abstract}
The light power transmitted by a diffusively illuminated slit of finite thickness is observed to depend stepwise on the slit width The steps have equal height and a width of one half the wavelength of the monochromatic light used This novel diffraction phenomenon is the analoguc of the quantization of the conductance of a point contact in a two-dimensional electron gas In contrast to the electronic case, absoiption at the walls of the slit plays an important role in determining the shape of the steps, as we show from a model calculation
\end{abstract}

\section{Introduction}

Diffraction of light by an aperture is an easily observed and widely known manifestation of the wave nature of light. As a direct consequence of this diffraction, the transmission cross-section $\sigma$ of an aperture for an incident plane wave differs from its geometrical area $A$. The relation between $\sigma$ and $A$ is a function sensitive to the detailed properties of the aperture [1-4].

Recently, it was pointed out that this relation is remarkably simplified for the case of diffuse (i.e. isotropic rather than plane-wave) illumination [5]. It was predicted that $\sigma$ increases with $A$ in a series of steps of equal height. A similar simplification occurs for two-dimensionally diffuse illumination of a slit, in a plane perpendicular to the slit. The transmission cross-section per unit length of the slit, $\sigma^{\prime}$, is predicted to increase stepwise as a function of its width $W$. The steps occur whenever $W=n \lambda / 2$, with $n=1,2,3, \ldots$, i.e. when a new mode is enabled in the slit. The diffuse illumination is required to couple equally to all modes [5].

The optical transmission characteristics of a slit have been studied extensively for plane wave illumination [6-9]. It was but recently, that the first observations of the discretised transmission cross-section for diffuse illumination were reported [10], analogously to the discretised con- ductance of a quantum point contact $[11,12]$. In this paper we summarise our findings and discuss some (not previously published) calculations on the influence of absorption on the shape of the transmission steps.

\section{The experiment}

We did the experiment at a wavelength of $1.55 \mu \mathrm{m}$. The set-up is presented schematically in fig. 1. The device consists of two halves of an integrating sphere ( $40 \mathrm{~mm}$ diameter) made of aluminum and coated with diffusively scattering barium sulfate. The slit is at the top of the sphere where the metal is only $25 \mu \mathrm{m}$ thick. Inside the slit, the aluminum is covered with silver to obtain a high reflection coefficient, which is required to avoid destruction of the transmission staircase by excessive absorption at the walls of the slit. The transmitted light was collected by the integrating sphere and detected. The slit width was varied by a piczo-electric transducer, and was monitored by a Michelson interferomcter.

The laser beam was expanded by a microscope objective and scattered by a diffusor. Diffuse illumination in two dimensions only (no propagation in the direction parallel to the slit) is crucial to the experiment since a slit rather than an 

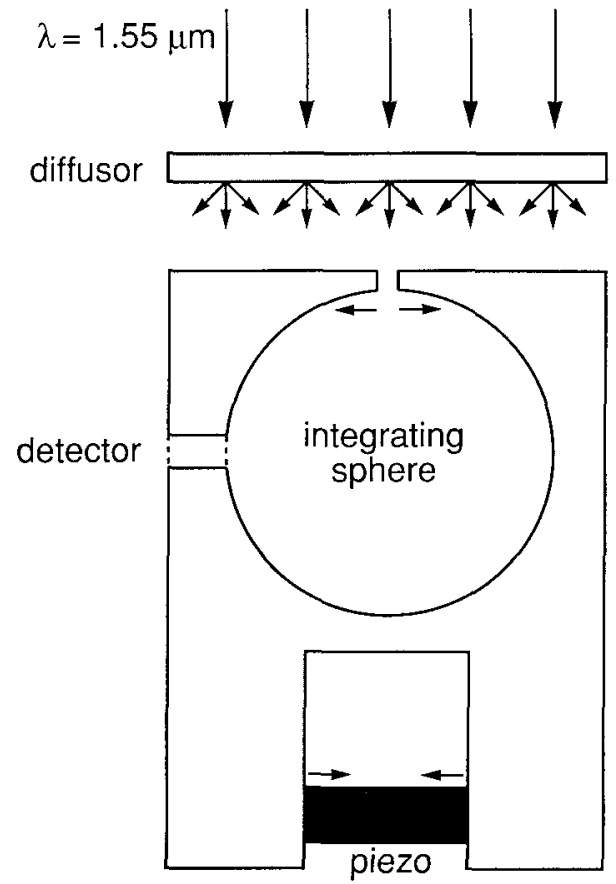

Fig 1 Schematic illustration of the set-up

aperture was used. Due to the large bandwidth of the laser $(15 \mathrm{~nm})$, the illumination was essentially incoherent.

The experimental results are presented in fig. 2 , which shows the transmitted power as a function of the slit width. Trace (a) was obtained

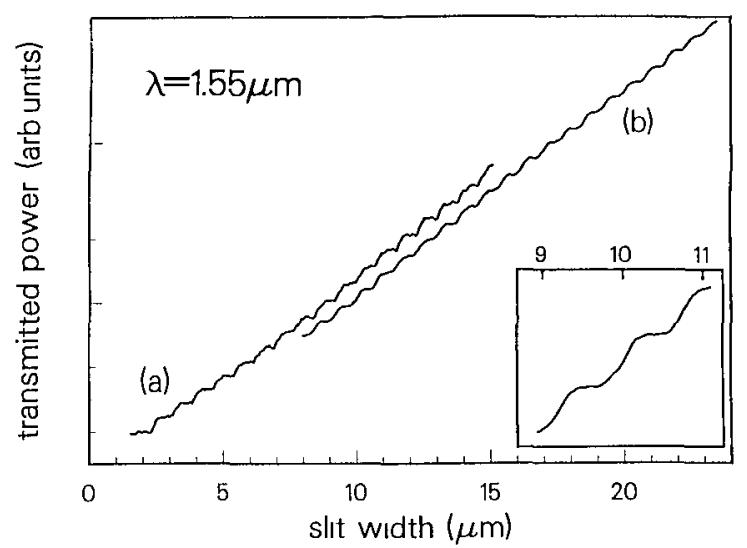

Fig 2. Transmitted power as a function of the shit width $W$, using a paper diffuser (a) and a glass-fibre diffuser (b), trace $b$ is scaled and shifted vertically for clarity The inset shows an enlarged part of trace $b$ using a paper diffusor and two slits in order to make the light diffusive in a plane only. Trace (b) was obtained using a diffusor made of very many parallel glass fibres [13]. Because the latter method is intrinsically two-dimensional, it produces a higher illumination intensity, and thus a better signal-to-noise ratio. A stepwise increase of the transmitted power is clearly observed in both traces. The steps occur at $\lambda / 2$ intervals in $W$, as predicted [5]. We also see that all steps have an equal height, implying that each mode transmits the same power. Because for large slit widths $(W / \lambda \rightarrow \infty) \sigma^{\prime}$ is equal to $W$, the steps in $\sigma^{\prime}$ must be equal to $\lambda / 2$, the size of the intervals in $W$.

\section{The shape of the steps}

The steps in the transmission cross-section are not abrupt. Partly, this is caused by non-uniformities in the slit width. Another cause is the slight absorption of radiation at the walls of the slit, which remains in spite of the use of a silver coating. The resulting damping of the propagating modes [13] causes a rounding of the steps and a slight curvature of the staircase for the first few steps visible in trace (a). Rounding of the steps is also partly due to non-adiabatic coupling (with inter-mode scattering) between the narrow slit and the infinite space [14].

The polarisation (the direction of the electric field) of the (two-dimensionally) diffusive light can be chosen to be either parallel (TE mode) or perpendicular (TM mode) to the direction of the slit. The attenuation of light in the slit for the TE and TM polarisation differs significantly. This absorption results from the penetration of the electric field in the (finitely conductive) metal. For a TM mode, the field perpendicular to the slit is constant, but for a TE mode the field is (in the ideal case) a sine (see fig. 3), and thus has much more field energy in the slit than in the conductors. Hence the attenuation of the TM modes will be much larger than for the TE modes.

The effect of absorption on the shape of the steps does not play a role in the electronic 


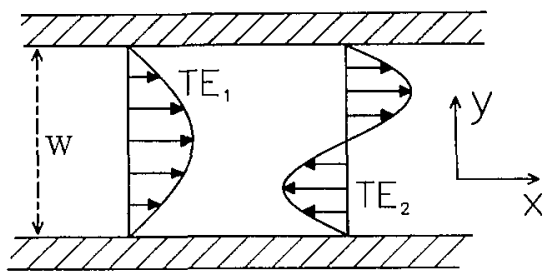

Fig 3 The mode profiles of the two lowest $\mathrm{TE}_{1}$ and $\mathrm{TE}$, modes in a perfectly conductıng parallel plate waveguide of width $W$ The solid arrows represent the electric field $L$ and the propagation of the wave is in the $z$ direction

counterpart, and has therefore not been investıgated previously To study this effect we will now calculate the attenuation of the TE modes in a lossless dielectric between two infinitely large conducting plates at a distance $W$, starting from Maxwell's equations [15] The wave equation is

$\nabla^{2} \boldsymbol{E}(x, y, z)=-\mu \varepsilon \omega^{2} \boldsymbol{E}(x, y, z)$,

which also holds for the magnetic field $\boldsymbol{H}$, and where the time dependence $\exp (1 \omega t)$ has already been accounted for by insertion of $1 \omega$ for the operator $\partial / \partial t$ We identify $\mu \varepsilon \omega^{2}=k^{2}=k_{\lambda}^{2}+$ $k_{y}^{2}+k_{z}^{2}$, with $k$ complex With $\varepsilon_{\mathrm{d}}$ and $\varepsilon_{\mathrm{c}}=\varepsilon_{\mathrm{c}}^{\prime}+$ $1 \varepsilon_{\mathrm{c}}^{\prime \prime}$ the permittivity of the dielectric and conductor, respectively, we have $\mu_{0} \varepsilon_{0} \varepsilon_{\mathrm{d}} \omega^{2} \equiv k_{\mathrm{d}}^{2}$ and $\mu_{0} \varepsilon_{0}\left(\varepsilon_{\mathrm{c}}^{\prime}+1 \varepsilon_{\mathrm{c}}^{\prime \prime}\right) \omega^{2} \equiv k_{\mathrm{c}}^{2}$

The propagation constants in the conductor and the dielectric should be matched, so $k_{a} \equiv$ $k_{z \mathrm{c}}=k_{z \mathrm{~d}}$, and because we use a plane wave propagating in the $z$-direction, $k_{\lambda_{\mathrm{c}}}=k_{\mathrm{x}_{\mathrm{d}}}=0$ We find that $k_{\mathrm{ic}}^{2}-k_{\mathrm{c}}^{2}=-k_{z}^{2}=-k_{y \mathrm{~d}}^{2}-k_{\mathrm{d}}^{2}$

After putting the proper boundary conditions on the interface of the conductors and dielectric at $y= \pm W / 2$ we eventually find

$\frac{ \pm k_{y \mathrm{~d}}^{2} \exp \left(-1 k_{y_{\mathrm{d}}} W\right)}{\left[1 \pm \exp \left(-1 k_{y_{\mathrm{d}}} W\right)\right]^{2}}=\mu_{0} \varepsilon_{0} \omega^{2}\left(\varepsilon_{\mathrm{c}}^{\prime}-\varepsilon_{\mathrm{d}}+1 \varepsilon_{\mathrm{c}}^{\prime \prime}\right)$,

where the \pm sign selects between the even and odd $\mathrm{TE}_{n}$ modes In a cavity without loss we find $k_{y \mathrm{~d}} W=n \pi$ If we now use that $k_{x \mathrm{~d}}^{2}+k_{y \mathrm{~d}}^{2}+$ $k_{z \mathrm{~d}}^{2} \equiv k_{\mathrm{d}}^{2}$, with $k_{x \mathrm{~d}}=0, k_{z \mathrm{~d}} \equiv k_{z} \equiv k_{z}^{\prime}+{ }_{1} k_{z}^{\prime \prime}$, and $k_{\mathrm{d}}^{2}=\varepsilon_{\mathrm{d}} k_{\mathrm{v} \mathrm{c}}^{2}$, we find the complex wave vector in

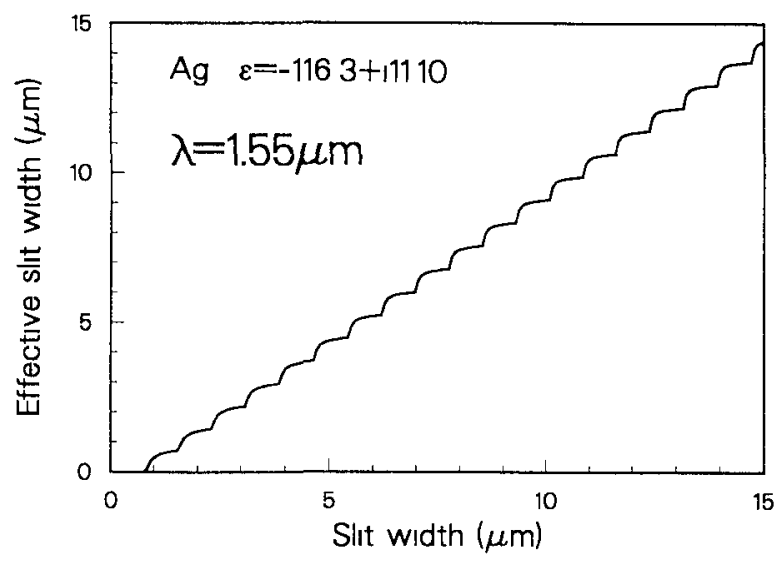

Fig 4 Calculated rounding of the steps due to absorption for a silver screen of thickness $L=25 \mu \mathrm{m}$ at a wavelength of $\lambda=155 \mu \mathrm{m}$

the propagation direction

$k_{z}^{\prime}+{ }_{1} k_{c}^{\prime \prime}=\sqrt{\varepsilon_{\mathrm{d}} k_{\mathrm{v} \text { lc }}^{2}-k_{y \mathrm{~d}}^{2}}$

The absorption for the intensity of the $n$th mode corresponding to this wave vector in a guide of length $L$ is then given by

$T_{n}=\exp \left(-2 k_{z}^{\prime \prime} L\right)$

In fig 4 the calculated total transmission $T=$ $\sum_{n} T_{n}$ is shown versus the width $W$ for the $\mathrm{TE}_{n}$ modes of a parallel-plane waveguide We used the dielectric constant of silver [16] at a wavelength $\lambda_{\mathrm{v} \text { ic }}=155 \mu \mathrm{m}$ It implicitly is assumed that the thickness of the plates $L=25 \mu \mathrm{m}$ is much larger than their mutual distance $W$, and that all modes were equally excited

The rounding of the steps visible in fig 4 is due entirely to absorption, since the rounding due to the intermode scattering at entrance and exit of the slit [14] has been neglected in this calculation

\section{Discussion}

In conclusion, we have reported the observation of the optical analogue of the conductance quantization of a point contact We calculated 
the rounding of the steps resulting from absorption in the case of TE polarisation

It is remarkable that this optical phenomenon, with its distinctly 19th century flavour, was not noticed prior to the discovery of its electronic counterpart There is an interesting parallel in the history of the discovery of the two phenomena In the electronic case, the Landauer formula

$$
G=\frac{e^{?}}{h} \sum_{n=1}^{N} T_{n}
$$

was already known before the quantised conductance of a point contact was discovered The reason that this discovery came as a surprise, wds that the relation $G=\left(e^{2} / h\right) N$ (following from the Landauer formuld for $T_{n}=1$ ) was regarded as an order of magnitude estimate [17] In order to have true quantisation, the relative error in this estımate must be smaller than $1 / N$, which at that time was not obvious

The equivalent of the Landauer formula in optics for the transport of electromagnetic modes has been known for a long time It is interesting to see that also in this ficld it was not noticed that the relation $T=N$ holds with a better than $1 / N$ accuracy This is particularly appdrent in, for example, a paper by Snyder and Pask [18], where they expect the relation $T \approx N$ to hold only in the geometrical optics limit, $\lambda \rightarrow 0$

\section{Acknowledgements}

We thank Professor J P Woerdman for drawing our attention to the optical equivalent of the Landauer formula We are grateful to $\mathrm{G} \mathrm{J} \mathrm{J}$ Geboers and A W Sleutjes for the fabrication of the slit Discussions with $\mathrm{Q} \mathrm{H} \mathrm{F}$ Vrehen and $\mathrm{H}$ van Houten were very valuable We would like to thank P J A Thiss for providing a high-power semiconductor laser

\section{References}

[1] C I Bouwkamp Rep Progr Phys 17 (1954) 35

[2] RWP King and T T Wu in The Scattenng and Diffraction of Waves (Harvard University Press Cim bridge 1959) p 113

[3] J J Bowman T B A Senıor and P L E Uslenghı Elec tromagnetic and Acoustic Scattering by Simple Shapes (North Holland Amstcrdam 1969)

[4] J D Jackson Classical Electrodynamics 2nd Ed (Wiley New York 1975)

[5] H van Houten and CWJ Beenakker in Analogies in Optics and Micro Electronics eds W van Haeringen and D Lenstra (Kluwer Dordrecht 1990) p 203

[6] S C Kashyap and M A K Hamid IEEE Tians on Antennas and Propagation 19 (1971) 499

[7] FL Neerhoff and G Mur Appl Sc1 Res 29 (1973) 73

[8] A Z Elshcrbcn and M Hamıd Can J Phys 65 (1987) 16

「9] M T Lightbody and M A Fiddy Adv Electron Elec tron Phys 19 (1987) 61

[10] E A Montie E C Cosman GW t Hooft M B van der Mark and CW J Becnakker Nature 350 (1991) 594

[11] B J van Wees $H$ van Houten CW J Beenakker J G Willamson L P Kouwenhoven D van der Marel and C T Foxon Phys Rev Lett 60 (1988) 848

[12] D A Wharam T J Thornton R Newbury M Pepper $H$ Ahmed J E F Frost D G Hasko D C Peacock D A Ritchic and G A C Jones J Phys C 21 (1988) L209

[13] M B van der Mark Thesıs University of Amsterdam (1990)

[14] A Szafer and A D Stone Phys Rev Lett 62 (1989) 300

[15] H A Atwater Introduction to Microwdve Theory (McGraw Hill 1962)

[16] G Hass and L Hadley Optical Propertics of Mctals American Instıtute of Physics Handbook 2nd Ed (McGraw Hill 1963) p 6

[17] Y Imry in Directions in Condensed Matter Physics eds $G$ Grinstem and $G$ Mazenko (World Scientific Singaporc 1986) p 130

[18] A W Snyder and C Pask J Opt Soc Am 63 (1973) 806 